\title{
SUMMABILITY OF SUBSEQUENCES
}

RALPH PALMER AGNEW

1. Introduction. Let $a_{n k}(n, k=1,2, \cdots)$ be a matrix of real or complex constants for which

$$
\begin{aligned}
& \lim _{n \rightarrow \infty} a_{n k}=0 \text {, } \\
& k=1,2,3, \cdots, \\
& \lim _{n \rightarrow \infty} \sum_{k=1}^{\infty} a_{n k}=1 ; \quad \sum_{k=1}^{\infty}\left|a_{n k}\right|<M, \quad n=1,2,3, \cdots,
\end{aligned}
$$

$M$ being a constant. This matrix defines a regular method of summability by means of which a sequence $x_{n}$ of real or complex numbers is summable to $X$ if $X_{n}=\sum_{k=1}^{\infty} a_{n k} x_{k}, n=1,2,3, \cdots$, exists and $\lim X_{n}=X$. It has recently been shown by R. C. Buck that if the sequence $x_{n}$ is real, bounded, and divergent, then the sequence has a subsequence not summable $A$. This note proves the following more general theorem.

Theorem. Let $A$ be regular and let $x_{n}$ be a bounded complex sequence. Then there exists a subsequence $y_{n}$ of $x_{n}$ such that the set $L_{Y}$ of limit points of the transform $Y_{n}$ of $y_{n}$ includes the set $L_{x}$ of limit points of the sequence $x_{n}$.

If $x_{n}$ is a bounded divergent sequence, then $L_{x}$ and hence also $L_{Y}$ must contain at least two distinct points and accordingly the subsequence $y_{n}$ is not summable $A$. Applying the theorem to the divergent sequence $0,1,0,1, \cdots$, we obtain the result of Steinhaus ${ }^{2}$ that there is a sequence of 0 's and 1 's not summable $A$.

2. Proof of the theorem. Let $L_{x}$ be the set of limit points of the bounded complex sequence $x_{n}$. Since the complex plane is separable and $L_{x}$ is a closed set, there is a countable (finite or infinite) subset $E$ of $L_{x}$ such that the closure $\bar{E}$ of $E$ is the set $L_{x}$ itself. Let $u_{1}, u_{2}, u_{3}, \ldots$ be a sequence containing all of the points of $E$; in case $E$ is a finite set, the points $u_{1}, u_{2}, u_{3}, \cdots$ are not distinct. Let the elements of the sequence

$$
u_{1} ; u_{1}, u_{2} ; u_{1}, u_{2}, u_{3} ; \cdots ; u_{1}, u_{2}, \cdots, u_{n} ; \cdots
$$

Presented to the Society, April 28, 1944 ; received by the editors February 5, 1944.

${ }^{1}$ R. C. Buck, A note on subsequences, Bull. Amer. Math. Soc. vol. 49 (1943) pp. 898-899.

${ }^{2} \mathrm{H}$. Steinhaus, Some remarks on the generalization of limit (in Polish), Prace Matematyczno-fizyczne vol. 22 (1911) pp. 121-134. 
be denoted by $v_{1}, v_{2}, v_{3}, \cdots$. The sequence $v_{n}$ is a sequence of points in $L_{x}$, and the set of limit points of the sequence is the set $L_{x}$. For each $p=1,2,3, \cdots$, let

$$
x\left(n_{p 1}\right), x\left(n_{p 2}\right), x\left(n_{p 3}\right), \cdots
$$

be a subsequence of $x_{n}$ having the limit $v_{p}$.

To simplify typography, we write $a(n, k)$ for $a_{n k}$. Since $A$ is regular, (1.1) and (1.2) hold. Hence sequences $n_{1}<n_{2}<n_{3}<\cdots$ and $k_{1}<k_{2}<k_{3}<\cdots$ of indices exist such that for each $p=1,2,3, \ldots$

$$
\sum_{k=1}^{k_{p}}\left|a\left(n_{p}, k\right)\right|<\frac{1}{p}, \quad \sum_{k=k_{p+1}}^{\infty}\left|a\left(n_{p}, k\right)\right|<\frac{1}{p} .
$$

It follows that

$$
\begin{aligned}
\sum_{k=k_{p}+1}^{k_{p+1}} a\left(n_{p}, k\right)= & \sum_{k=1}^{\infty} a\left(n_{p}, k\right)-\sum_{k=1}^{k_{p}} a\left(n_{p}, k\right) \\
& -\sum_{k=k_{p+1}+1}^{\infty} a\left(n_{p}, k\right)=1+\epsilon_{p}
\end{aligned}
$$

where, here and hereafter, $\epsilon_{p}$ denotes generically a sequence for which $\epsilon_{p} \rightarrow 0$ as $p \rightarrow \infty$.

The subsequence $y(n)$ of the given sequence $x_{n}$ is now selected as follows. Assuming that, for a fixed index $p, y(k)$ has been selected for each $k \leqq k_{p}$, let $y\left(k_{p}+1\right), \cdots, y\left(k_{p+1}\right)$ be selected from the sequence (2.2) in such a way that $y(j)$ is a predecessor of $y(k)$ in the sequence $x_{n}$ when $j<k$ and

$$
\left|y(k)-v_{p}\right|<1 / p, \quad k_{p}<k \leqq k_{p+1} .
$$

Since $x_{n}$ is bounded, say $\left|x_{n}\right| \leqq B$, the subsequence $y(n)$ thus defined by induction is bounded and accordingly possesses a transform

$$
Y_{n}=\sum_{k=1}^{\infty} a_{n, k} y_{k}
$$

For each $p=1,2,3, \cdots$

$$
\begin{aligned}
Y\left(n_{p}\right) & =\sum_{k=1}^{k_{p}} a\left(n_{p}, k\right) y_{k}+\sum_{k=k_{p+1}+1}^{\infty} a\left(n_{p}, k\right) y_{k}+\sum_{k=k_{p}+1}^{k_{p+1}} a\left(n_{p}, k\right) y_{k} \\
& =\epsilon_{p}+\sum_{k=k_{p}+1}^{k_{p+1}} a\left(n_{p}, k\right) y_{k}
\end{aligned}
$$


since each of the first two terms of the second member of (2.7) is dominated by $B / p$. Moreover

$$
\begin{aligned}
\sum_{k=k_{p}+1}^{k_{p+1}} a\left(n_{p}, k\right) y_{k} & =v_{p} \sum_{k=k_{p}+1}^{k_{p+1}} a\left(n_{p}, k\right)+\sum_{k=k_{p}+1}^{k_{p+1}} a\left(n_{p}, k\right)\left(y_{k}-v_{p}\right) \\
& =v_{p}\left(1+\epsilon_{p}\right)+\epsilon_{p}=v_{p}+\epsilon_{p} .
\end{aligned}
$$

Therefore the sequences $Y\left(n_{p}\right)$ and $v_{p}$ have the same limit points and accordingly the set of limit points of the sequence $Y\left(n_{p}\right)$ is identical with the set $L_{x}$ of limit points of the sequence $x_{n}$. The set $L_{Y}$ of limit points of the complete sequence $Y_{n}$ therefore includes the set $L_{x}$ and the theorem is proved.

3. Conclusion. It is apparent from the proof of the theorem that if $x_{n}$ is a bounded divergent sequence, then it is possible to construct many subsequences $y_{n}$ not summable $A$. However, the class of such subsequences $y_{n}$ thus constructed seems to be a "small" subclass of the class of all subsequences of $x_{n}$. This observation is in agreement with the fact, recently proved by Buck and Pollard, ${ }^{3}$ that if $A$ is either convergence or the Cesàro method of order 1 and $s_{n}$ is a real bounded sequence summable $A$, then there is a sense in which "almost all" of the subsequences of $x_{n}$ are summable $A$.

The theorem states that the set $L_{Y}$ of limit points of the transform $Y_{n}$ of the subsequence $y_{n}$ of $x_{n}$ includes the set $L_{x}$ of limit points of $x_{n}$. In some cases, $L_{Y}$ is identical with $L_{x}$. This is so when $A$ is convergence. In some cases, $L_{Y}$ is more extensive than $L_{x}$. This is so when $A$ is a Cesàro method of positive order and the set $L_{x}$ is not connected since, as was shown by Barone, ${ }^{4}$ the set of limit points of the transform of each bounded sequence must be connected. The same is true for methods of Hölder, Riesz, de la Vallee Poussin, and Euler.

\section{CoRnell UNIVERsity}

${ }^{3}$ R. C. Buck and Harry Pollard, Convergence and summability properties of subsequences, Bull. Amer. Math. Soc. vol. 49 (1943) pp. 924-931.

${ }^{4} \mathrm{H}$. G. Barone, Limit points of subsequences and their transforms by methods of summability, Duke Math. J. vol. 5 (1939) pp. 740-752. 